\title{
Prevalencia y factores asociados al consumo de marihuana en estudiantes de 18 a 25 años de una universidad pública, Colombia
}

\author{
Prevalence and associated factors to marijuana consumption in students between 18 to 25 years old from a \\ Colombia's public university
}

\author{
Javier Martínez-Torres', Florencio Arias-Coronel2, Abner Enrique Rodelo-Vega ${ }^{3}$, Neydis Patricia Jaraba-Toro4, Leyla \\ Mariela Meza-Castellanos ${ }^{5}$, Magda Milena Contreras-Jáureguib, Sandra Padilla-Sarmiento7, Diana Janeth Villamizar- \\ Carrillo $8^{*}$
}

1 Estudiante Doctorado en epidemiología, Magíster Epidemiología. Grupo GICAEDS. Facultad de Cultura Física, Deporte y Recreación. Universidad Santo Tomás. Bogotá, Colombia. e-mail: javiermartinezł@usantotomas.edu.co

2 Magíster en Neurorehabilitación. Universidad María Cano. Medellín, Colombia. e-mail: Florencioariascoronel@hotmail.com

3 Terapeuta ocupacional. Universidad de Pamplona. Colombia. e-mail: Abner.R129@hotmail.com

4 Terapeuta ocupacional. Universidad de Pamplona. Colombia. e-mail: Jaraba031291@hotmail.com

5 Terapeuta ocupacional. Universidad de Pamplona. Colombia. e-mail: Meza.Leyla92@hotmail.com

6 Magíster en Orientación Vocacional, Estudiante Doctorado Educación. Grupo "DIDOH" Departamento de Terapia Ocupacional, Universidad de Pamplona. Pamplona, Colombia.e-mail: dto@unipamplona.edu.co

7 Magíster en educación. Grupo de investigación "Psicología y sociedad", Programa de Psicología Universidad de Pamplona. Pamplona, Colombia. e-mail: spadilla@unipamplona.edu.co

8 PhD. en Psicología. Grupo de Investigación "Psicología y Sociedad", Programa de Psicología, Universidad de Pamplona. Pamplona, Colombia. e-mail: jacarrillo71@unipamplona.edu.co

Martínez-Torres J, Arias-Coronel F, Rodelo-Vega AE, Jaraba-Toro NP, Meza-Castellanos LM, Contreras-Jáuregui MM, PadillaSarmiento SM, Villamizar-Carrillo DJ. Prevalencia y factores asociados al consumo de marihuana en estudiantes de 18 a 25 años de una Universidad Pública, en Norte de Santander-Colombia, durante el primer semestre de 2014. Univ. Salud. 2016;18(3):525-531. DOI: http://dx.doi.org/10.22267/rus.161803.57

\begin{abstract}
Resumen
Introducción: El consumo de drogas, en general, se ha extendido alrededor de todo el mundo y por ende, se considera un problema grave de salud pública, que le concierne a los sistemas de salud, puesto que estas son unas sustancias que producen adicción; ello conlleva el deterioro cognitivo, con mayor énfasis en memoria, aprendizaje y también pérdida del control interno. Objetivo: Determinar la prevalencia y factores asociados al consumo de marihuana durante el último mes y alguna vez en la vida, en estudiantes universitarios colombianos de una universidad pública entre 18 y 25 años de edad. Materiales y métodos: Estudio descriptivo, transversal, en 474 sujetos sanos entre los 18 y 25 años, de Pamplona, Colombia. Los factores asociados al consumo de marihuana (género, creencia religiosa, semestre académico, estrato, edad, consumo de cigarrillo y consumo de alcohol), se recogieron por encuesta estructurada. Se calcularon prevalencias de cada factor y se establecieron asociaciones mediante la construcción de modelos de regresión. Resultados: Se encontró al momento de la encuesta que el 7,1\% de los estudiantes habían consumido marihuana en el último mes, y un $22 \%$ manifestaron haberla usado al menos una vez. Los modelos de regresión muestran que tener el hábito tabáquico se asoció con el uso de marihuana (OR 23,33 IC95\% 6,92-78,68). Conclusión: El consumo de marihuana en la población estudiada fue alta y factores como el consumo de cigarrillos se encuentran asociados con el de marihuana. Estos datos podrían utilizarse para ofrecer programas de intervención educativa.
\end{abstract}


Palabras clave: Cannabis; adulto joven; prevalencia; hábito de fumar marihuana; Colombia; estudios transversales. (Fuente: DeCS, Bireme).

\begin{abstract}
Introduction: Drug use in general has spread around the world and, therefore, it is considered to be a serious public health problem that concerns health systems, since they are substances that produce addiction and entails cognitive impairment with greater emphasis on memory, learning and loss of internal control. Objective: To determine prevalence and associated factors to marijuana consumption during the last month and once in a lifetime in public university students in Colombia who are between 18 to 25 years old. Materials and methods: A descriptive cross sectional study was performed in 474 healthy subjects between 18 to 25 years old in Pamplona, Colombia. The associated factors to marijuana consumption (gender, religious belief, academic semester, social status, age, smoking and alcohol consumption.), were collected by a structured survey. Prevalence for each factor was calculated and associated factors were established through the construction of regression models. Results: At the time of the survey, it was found that $7.1 \%$ of students smoked marijuana in the last month and $22 \%$ expressed having used it at least once. The regression models show that having the habit of smoking cigarette was associated to marijuana consumption (OR 23.33 CI95\% 6.92-78.68). Conclusion: Marijuana use in the study population was high and factors such as the consumption of cigarettes are associated with that of marijuana. These data could be used to provide educational intervention programs.
\end{abstract}

Keywords: Cannabis; young adult; prevalence; marijuana smoking; Colombia; cross-sectional studies. (Source: DeCS, Bireme).

\section{Introducción}

La cafeína, la nicotina, el etanol y la marihuana (tetrahidrocannabinol THC) se encuentran entre las sustancias adictivas más comunes y culturalmente, aceptados en la sociedad occidental $^{1}$. La menos aceptada de dicho grupo es la última de las citadas, debido a un estigma social que relaciona su consumo con actividades delictivas $^{2}$; esa es una sustancia psicoactiva ampliamente usada en la sociedad Europea y Americana, especialmente, entre los más jóvenes ${ }^{3}$.

El consumo de drogas, en general, se ha extendido alrededor de todo el mundo $y$, por ende, se considera un problema grave de salud pública, que le concierne a los sistemas de salud ${ }^{4}$, puesto que estas son sustancias que producen adicción; ello conlleva el deterioro cognitivo, con mayor énfasis en memoria, aprendizaje y también pérdida del control interno ${ }^{5}$. Estudios demuestran los efectos deletéreos que tiene el consumo de marihuana. Desde el punto de vista social, se encuentra el desinterés por ser parte de un grupo social funcional ${ }^{6}$ y aumenta la probabilidad de un accidente de tránsito ${ }^{7}$, desde el punto de vista psicológico, se relaciona con trastornos afectivos, de ansiedad, de conducta, de la conducta alimentaria; psicóticos por déficit de atención con hiperactividad ${ }^{3,8-10}$ y desde lo fisiológico, cambios inflamatorios y favorece la aparición de lesiones pre-cancerosas en las vías respiratorias, las cuales son proporcionales a la magnitud del consumo ${ }^{7}$.

La llegada a la universidad del adolescente está ligada a la adaptación a nuevos estilos de vida, y a modificación en los círculos sociales y redes de apoyo, que conducen a la adquisición de patrones diferentes de comportamiento, los cuales se pueden ver influenciados por los pares, la soledad o el estrés que requiere el esfuerzo para superar las asignaturas; dicha maraña adaptativa genera una vulnerabilidad en el adolescente y el adulto joven, que puede llevar a que ellos se inicien en el consumo de sustancias psicoactivas, tales como la marihuana, o drogas más densas ${ }^{8,11}$.

El estudio examinó la prevalencia y los factores sociodemográficos asociado al consumo de marihuana en su vida o durante el último mes previo al estudio, en una muestra de estudiantes universitarios colombianos, de Pamplona, en Norte de Santander, durante el primer semestre de 2014. 


\section{Materiales y métodos}

\section{Diseño del estudio, población y muestra}

Durante el primer periodo del 2014 se realizó un estudio de tipo descriptivo transversal, en la ciudad de Pamplona, Norte de Santander, Colombia evaluó una muestra proveniente de la población estudiantil de la Universidad pública de Norte de Santander. Para el cálculo del tamaño de la muestra se usaron los supuestos de una población de 7853 estudiantes matriculados al momento de la realización del estudio; la frecuencia esperada era del $50 \%$, con un error estándar del 5,0\% y un intervalo de confianza del 95\%. Para evitar un problema de no respuesta, se aumentó el valor de la muestra en un $20 \%$; la muestra a conveniencia involucró a 474 personas. Se reclutaron individuos que aseveraran estar matriculados en cualquiera de los programas y afirmaron ser mayor edad (según legislación colombiana). Todos los sujetos debían firmar el consentimiento informado, aceptar ser parte de la investigación. Se excluyeron a los menores de 18 años y los mayores de 25 años.

\section{Procesos de medición y recolección de la información}

Se realizó una encuesta estructurada de medición transversal, para evaluar el consumo de marihuana actual (consumo durante los últimos 30 días) y el haberla consumido alguna vez en la vida (lo cual será llamada de ahora en adelante: «haber probado marihuana»). La valoración del consumo actual de marihuana, o de haberla probado, se hizo por medio de una sola pregunta para cada caso, en la cual el sujeto acusaba con un sí o un no; para el consumo actual se preguntó si había fumado marihuana durante los últimos treinta días y para «haberla probado», se preguntó si había probado la marihuana alguna vez en la vida.

Para el presente análisis se definieron siete variables sociodemográficas: 1) sexo (masculino o femenino); 2) creencia religiosa (cristiano católico, cristiano no católico, ateo, otro); 3) semestre académico (entre I y II semestre; entre III y V semestre, superior a VI semestre); 4) estrato social (estrato social I y II; y estrato social III y IV); 5) edad (menor de 19 años; entre 20 y 21 años; mayor de 22 años); 6) consumo de cigarrillos en el último mes (sí, no); 7) consumo de alcohol en el último mes (sí, no). Para la edad se le pidió al sujeto que refiriese su edad en años cumplidos a la fecha, posterior a esto se categorizó en tres grupos. La encuesta fue aplicada de modo completamente anónimo y autodiligenciable.

\section{Análisis estadístico}

Se inició, con un análisis exploratorio para determinar la distribución de frecuencias (medidas de tendencia central y de dispersión para variables cuantitativas), frecuencias relativas (para variables cualitativas). Para estimar la relación entre el consumo actual de marihuana y aquellas que la probaron alguna vez, con las variables estudiadas se utilizaron dos modelos de regresión logística binaria para cada caso. El modelo de regresión logística binaria simple (modelo uno); el segundo, el cual se ajustó por todas las variables sociodemográficas (modelo dos). Los modelos de regresión fueron calculados con intervalos de confianza del $95 \%$. Los análisis fueron realizados con el programa estadístico SPSS, versión 20.

\section{Consideraciones éticas}

El estudio se desarrolló de acuerdo a las normas establecidas en la Resolución 8430 de 1993 del Ministerio de Salud, artículo 11, este estudio se clasifica como una investigación "sin riesgo". La participación fue voluntaria y quienes aceptaron formar parte del estudio firmaron un consentimiento informado. Los datos fueron manejados con estricta confidencialidad y anonimato sin realizarse análisis individuales, el formato de valoración estaba enumerado con un código el cual sólo fue útil para el proceso de tabulación y el cual estaba separado del registro de consentimiento informado.

\section{Resultados}

De las 474 personas incluidas, se analizaron 423 estudiantes (10.8\% perdidas) que completaron correctamente el cuestionario, cuyas edades oscilaban entre 18 y 25 años (edad media 20,3I C95 \%= 20,1-20,5). Se encontró una prevalencia 
de consumo actual de marihuana de $7.1 \%$ (IC95\%= 4,7\% - 9,1\%), mientras que para el caso de «haberla probado», el porcentaje que se estimó fue de $22,0 \%$ (IC95\%= 18,0\% - 26,0\%).

Los estudiantes pertenecientes al grupo masculino, ateo y quienes acusaron consumir cigarrillos presentaron los mayores porcentajes para consumo actual (8,7\%, 26,1\% y 20,8\%), respectivamente; la misma tendencia se evidenció en aquellos que han probado la marihuana $\quad(25,9 \%, \quad 56,5 \%, \quad 50,8 \%)$, respectivamente. Las demás características sociodemográficas se presentan en la tabla 1.

Tabla 1. Prevalencia de consumo de marihuana por variables sociodemográficas

\begin{tabular}{|c|c|c|c|c|c|c|c|c|}
\hline \multirow[b]{4}{*}{ Total } & \multicolumn{4}{|c|}{ Consumo en el último mes } & \multicolumn{4}{|c|}{ Consumo de vida } \\
\hline & \multicolumn{2}{|c|}{ Sí } & \multicolumn{2}{|c|}{ No } & \multicolumn{2}{|c|}{ Sí } & \multicolumn{2}{|c|}{ No } \\
\hline & $\mathbf{n}$ & $\%$ & $\mathbf{n}$ & $\%$ & $\mathbf{n}$ & $\%$ & $\mathbf{n}$ & $\%$ \\
\hline & 30 & $7.1 \%$ & 393 & 92.9 & 93 & 22.0 & 330 & 78.0 \\
\hline \multicolumn{9}{|l|}{ Género } \\
\hline Hombre & 23 & 8,7 & 240 & 91,3 & 68 & 25,9 & 195 & 74,1 \\
\hline Mujer & 7 & 4,4 & 153 & 95,6 & 25 & 15,6 & 135 & 84,4 \\
\hline \multicolumn{9}{|l|}{ Religión } \\
\hline Cristiano (católico) & 17 & 5,6 & 287 & 94,4 & 56 & 18,4 & 248 & 81,6 \\
\hline Cristiano (no católico) & 4 & 5,6 & 68 & 94,4 & 14 & 19,4 & 58 & 80,6 \\
\hline Ateo & 6 & 26,1 & 17 & 73,9 & 13 & 56,5 & 10 & 43,5 \\
\hline Otro & 3 & 12,5 & 21 & 87,5 & 10 & 41,7 & 14 & 58,3 \\
\hline \multicolumn{9}{|l|}{ Semestre } \\
\hline Entre 1 y 2 semestre & 12 & 5,5 & 205 & 94,5 & 38 & 17,5 & 179 & 82,5 \\
\hline Entre 3 y 5 semestre & 12 & 10,4 & 103 & 89,6 & 29 & 25,2 & 86 & 74,8 \\
\hline Superior a sexto semestre & 6 & 6,6 & 85 & 93,4 & 26 & 28,6 & 65 & 71,4 \\
\hline \multicolumn{9}{|l|}{ Estrato social } \\
\hline Estrato I y II & 22 & 7,3 & 278 & 92,7 & 58 & 19,3 & 242 & 80,7 \\
\hline Estrato III y IV & 8 & 6,5 & 115 & 93,5 & 35 & 28,5 & 88 & 71,5 \\
\hline \multicolumn{9}{|l|}{ Edad } \\
\hline Menor a 19 años & 7 & 4,1 & 164 & 95,9 & 24 & 14,0 & 147 & 86,0 \\
\hline Entre 20 y 21 años & 16 & 12,2 & 115 & 87,8 & 39 & 29,8 & 92 & 70,2 \\
\hline Superior a 22 años & 7 & 5,8 & 114 & 94,2 & 30 & 24,8 & 91 & 75,6 \\
\hline \multicolumn{9}{|c|}{ Consumo de cigarrillo en el último mes } \\
\hline Sí & 25 & $20,8 \%$ & 95 & 79,2 & 61 & 50,8 & 59 & 49,2 \\
\hline No & 5 & $1,7 \%$ & 298 & 98,3 & 32 & 10,6 & 271 & 89,4 \\
\hline \multicolumn{9}{|c|}{ Consumo de alcohol en el último mes } \\
\hline Sí & 22 & $8,7 \%$ & 231 & 91,3 & 76 & 30,0 & 177 & 70,0 \\
\hline No & 8 & $4,7 \%$ & 162 & 95,3 & 17 & 10,0 & 153 & 90,0 \\
\hline
\end{tabular}

En la figura 1, se presentan los resultados del análisis de regresión logística simple y múltiple, tras realizar el ajuste de tener entre 20 y 21 años (OR 3.05 IC95\% 1.07-8.71) y ser consumidor de cigarrillos (OR 23.33 IC95\% 6.92-78.68), se asociaron como factores para presentar el consumo actual. 
Figura 1. Análisis de regresión logística simple y múltiple que asocian factores que predisponen el consumo actual de marihuana
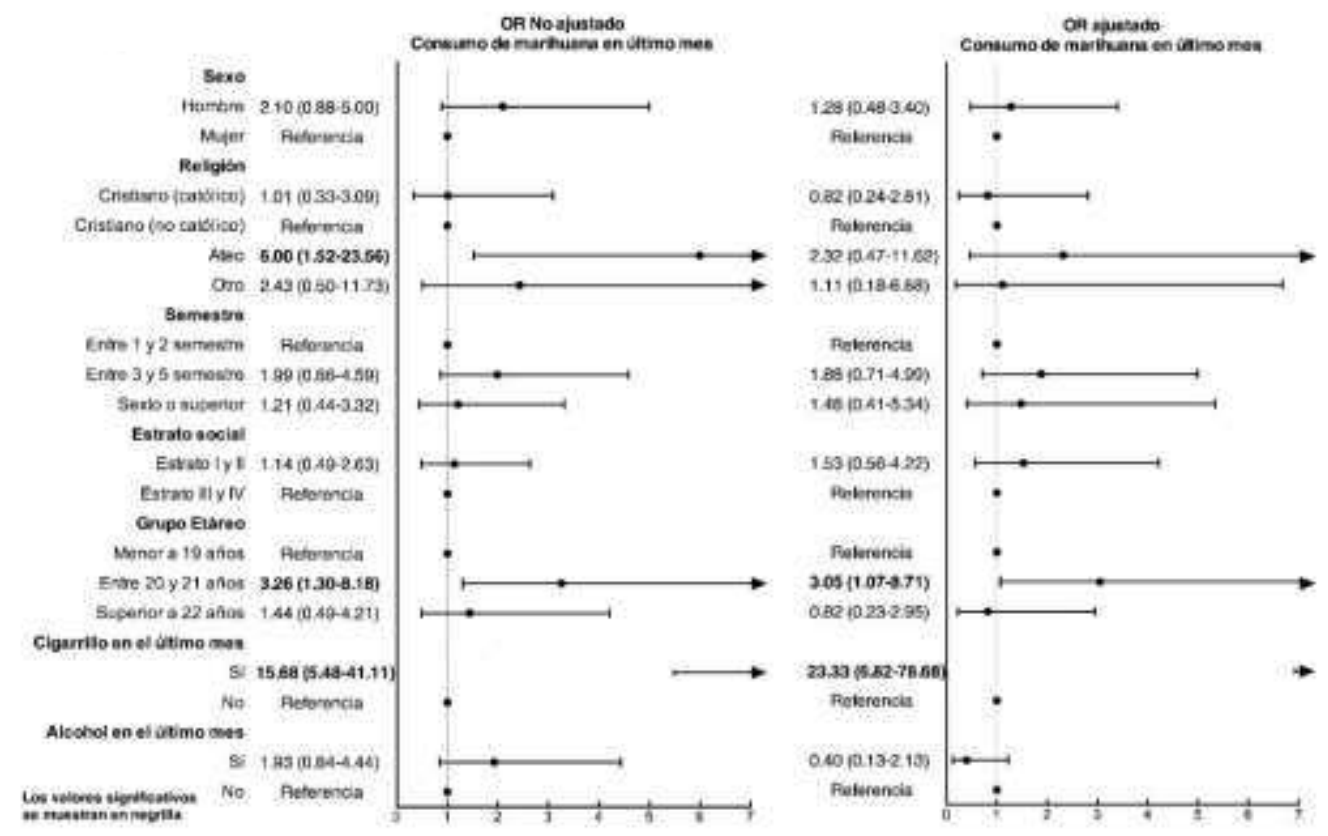

En la Figura 2, se presentan los resultados del entre 20 y 21 años (OR 2.33 IC95\% 1.18-4.59) y análisis de regresión logística simple y múltiple, para determinar la asociación entre el «haber probado marihuana» y las variables sociodemográficas. Al realizar los ajustes de edad reconocerse como fumador (OR 6.95 IC95\% 3.8412.60), se juntaron como factores asociados para «haber consumido marihuana» al menos una vez en la vida.

Figura 2. Análisis de regresión logística simple y múltiple que determinan la asociación entre variables sociodemográficas y el consumo de marihuana
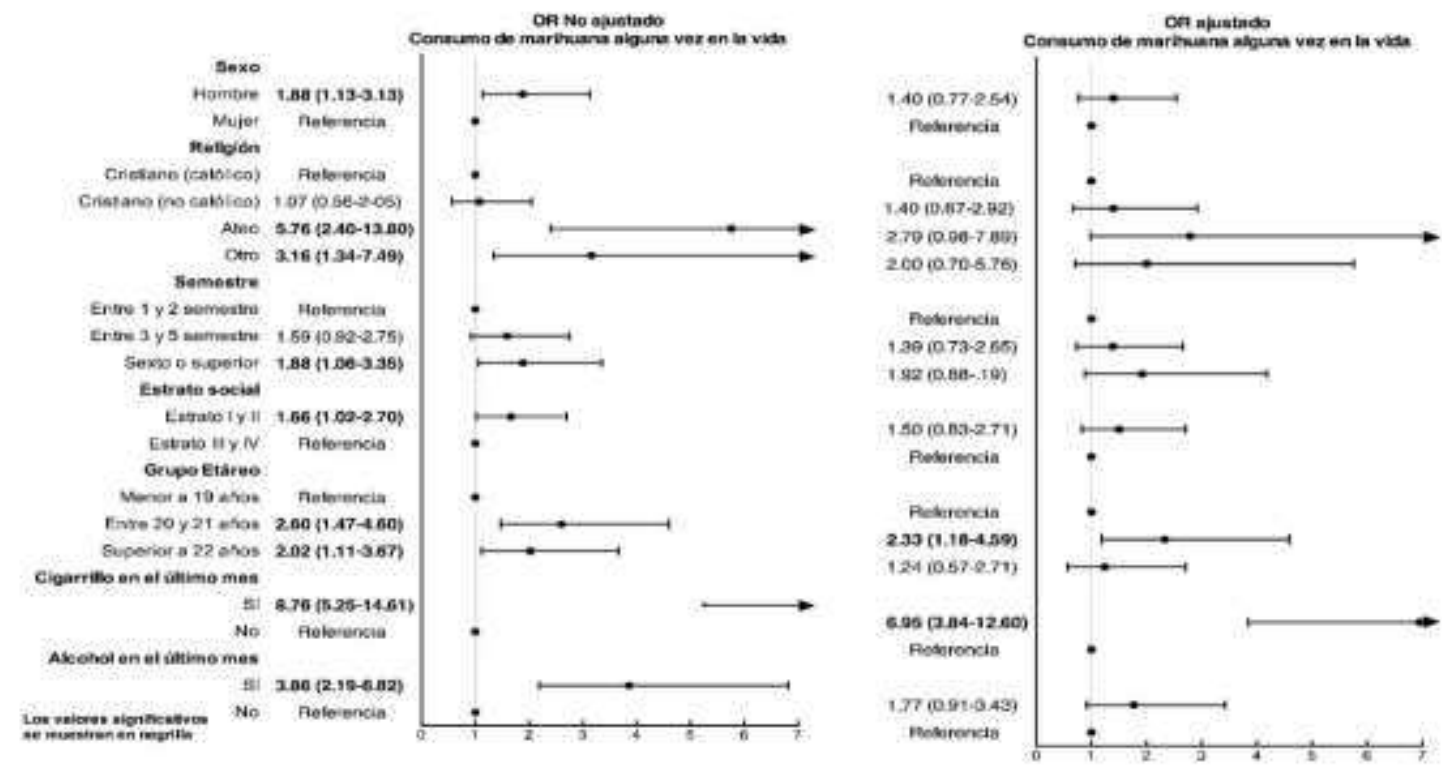


\section{Discusión}

El principal hallazgo de este estudio fue la prevalencia del $7,1 \%$ en el consumo actual de marihuana, resultado similar al descubierto por Medina et al ${ }^{11}$ en el año 2009 , quien encontró que en estudiantes universitarios de la ciudad de Bogotá el 8,2\% consumía marihuana; Otro estudio con estudiantes universitarios desarrollado en cuatro países, muestra que las prevalencia de consumo en el último año fue de $7,3 \%$ en colombianos, la cifra más alta de los cuatro países estudiados ${ }^{12}$. La prevalencia de consumo en el último mes de hombres fue aproximadamente dos veces superior al de mujeres $\quad(8,7 \%$ y $4,4 \%$ respectivamente). Tendencia similar se encontró para el consumo de alguna vez en la vida $(25,9 \%$ y $15,6 \%)$. Esos resultados concuerdan con la mayoría de estudios en población universitaria ${ }^{4,12,14}$.

Se ha descrito que las creencias religiosas pueden modificar o determinar estilos de vida, indicando que esa característica podría influir significativamente en las conductas de las personas ${ }^{16}$. La aseveración resulta válida para el presente estudio. Las mayores prevalencias del consumo de marihuana en el último mes con respecto a la creencia religiosa, se dio en las personas que acusaron ser ateas $(26,1 \%)$, mientras que para los cristianos fue casi una quinta parte $(5,6 \%)$. Resultados similares a un estudio realizado en jóvenes universitarios de Cartagena en el año 2011, muestran que aquellas personas que se sienten identificadas con el cristianismo tienen menor probabilidad de consumir sustancias psicoactivas, legales e ilegales, como cigarrillos, alcohol, marihuana y cocaína ${ }^{13}$.

Como seres sociales los humanos se encuentran en continuo cambio. Las personas, con el paso de los años, modifican sus creencias y sus contextos; junto con ellos se transforman los procesos sociales y los estilos de vida. En el presente estudio se encontró que el consumo de marihuana se asociaba con un grupo específico de edad; resultados similares a los de Quimbayo et al. ${ }^{4}$, en Bogotá en el 2013, donde se encontró que personas con edades comprendidas entre 18 y 21 años eran quienes más consumían ese tipo de sustancias.

La situación socioeconómica de los jóvenes es una consecuencia de medidas tales como el ingreso monetario de los padres o su ocupación, nivel académico de los padres y el acceso a los recursos; se ha descrito que los grupos sociales de los niveles más bajos se encuentran asociados con mayores tasas de prevalencia de diferentes eventos deletéreos ${ }^{17}$. Este hecho se ratifica en el presente estudio, puesto que la mayor prevalencia de consumo en el último mes se dio en los estratos 1 y 2 , resultados que concuerdan con los hallados por otros investigadores ${ }^{4,18}$.

El consumo de cigarrillos es considerado hoy el problema más grande de salud pública prevenible en países desarrollados ${ }^{19}$. Los resultados muestran que aproximadamente uno de cada cinco consumidores de cigarrillos consumen marihuana, y uno de cada dos la ha probado; estos resultados se encuentran relacionados con los descritos por otros autores, donde se asegura que el consumo de cigarrillos es una precuela para adentrarse en el consumo de drogas más fuertes ${ }^{20}$.

El alcohol es la droga con mayor aceptación social. Un estudio realizado con estudiantes de Cartagena mostró que tener problemas de consumo de alcohol está asociado con el consumo de marihuana ${ }^{13}$. En el presente estudio la prevalencia de consumo en el último mes, fue mayor para las personas que acusaron haber ingerido alcohol en los últimos treinta días.

Teniendo en cuenta el carácter descriptivo transversal de este estudio, las conclusiones respecto al modelo de asociación no permiten establecer una relación de causalidad, sino únicamente una relación de asociación. Sin embargo, eso no le resta validez a los hallazgos hechos ya que ellos reflejan una parte de la realidad social.

\section{Conclusión}

El consumo de marihuana en la población estudiada fue similar a la hallada en otros 
estudios, y factores como el consumo de cigarrillos se encuentran asociados con el de marihuana. Estos datos podrían utilizarse para ofrecer programas de intervención educativa, y para la generación de estrategias que se deben usar para contrarrestar la iniciación y perpetuación de consumo de sustancias psicoactivas.

\section{Limitaciones}

Tipo de muestreo.

\section{Agradecimientos}

Los autores agradecen a la Universidad de Pamplona, especialmente al decano de la Facultad de Salud, Henry Becerra por el apoyo brindado al proceso de investigación.

\section{Conflicto de intereses}

Los autores declaran no tener ningún conflicto de intereses.

\section{Referencias}

1. Pesta DH, Angadi SS, Burtscher M, Roberts CK. The effects of caffeine, nicotine, ethanol, and tetrahydrocannabinol on exercise performance. Nutr Metab (Lond). 2013;10(1):71-85.

2. Acero-Gonzalez A, Escobar-Cordoba C, CastellanosCastaneda G. Factores de riesgo para violencia y homicidio juvenil. Rev colomb psiquiatr. 2007;23(1): 78-97.

3. Torres G, Fiestas F. Efectos de la marihuana en la cognición: una revisión desde la perspectiva neurobiológica. Rev Peru Med Exp Salud Pública. 2012; 29(1):127-34.

4. Quimbayo-Díaz J, Olivella-Fernández M. Consumo de marihuana en estudiantes de una universidad colombiana. Rev. salud pública. 2013;15(1):32-43.

5. Vélez-García A, Ostrosky-Solís F, Borja-Jiménez K. Efectos del consumo de marihuana sobre la toma de decisión. Revista Mexicana de Psicología. 2010;27(2):309-315.

6. Kohn L, Kittel F, Piette D. Peer, family integration and other determinants of cannabis use among teenagers. Int J Adolesc Med Health. 2004;16(4):359-70.

7. Kalant H. Adverse effects of cannabis on health: an update of the literature since 1996. Prog Neuropsychopharmacol Biol Psychiatry. 2004;28(5): 849-63.

8. Moore T, Zammit S, Lingford-Hughes A, Barnes T, Jones $\mathrm{P}$, Burke M, et al. Cannabis use and risk of psychotic or affective mental health outcomes: a systematic review. Lancet. 2007;370(9584):319-28.
9. Potvin S, Amar M. Review: Cannabis use increases the risk of psychotic outcomes. Evid Based Ment Health. 2008;11(1):28.

10. Matalí-Costa J, Pardo-Gallego M, Trenchs-Sainz de la Maza V, Serrano-Troncoso E, Gabaldon Fraile S, Luaces Cubells C. Consumo de drogas en adolescentes. Dilema ético en el abordaje diagnóstico-terapéutico. Anales de Pediatría. 2009;70(4):386-90.

11. Medina-Matallana L, Cunningham J, Strike C, Brands B, Wright M. Normas percibidas por los estudiantes universitarios acerca de sus pares y el uso de drogas en Bogotá, Colombia. Rev Latino-am Enfermagem. 2009; 17(Esp):893-9.

12. Comunidad Andina. Estudio epidemiológico andino sobre consumo de drogas sintéticas en la población universitaria. Informe Corporativo: Bolivia, Colombia, Ecuador y Perú. [Online]. Perú; 2010 [cited 2015 Mayo 1. Disponible en: http://www.comunidadandina.org/public/Estudio_dro gas.pdf.

13. Cogollo-Milanés Z, Arrieta-Vergara K, Blanco-Bayuelo S, Ramos-Martínez L, Zapata K, Rodríguez-Berrio Y. Factores psicosociales asociados al consumo de sustancias en estudiantes de una universidad pública. Rev. salud pública. 2011;13(3):470-9.

14. Dirección de Bienestar Universitario Universidad de Antioquia. Universidad de Antioquia. [Online]:; 2013 [cited 2015 Abril 25. Disponible en: http://www.udea.edu.co/portal/page/portal/Bibliotec aProgramas/bienestarUniversitario/diseno/document os/Informe $\% 20$ PRADICAN $\% 20$ Universidad $\% 20 \mathrm{de} \% 2$ 0Antioquia.pdf

15. Martínez-Torres J, Perez-Buelvas J, Buchelli L, Manrique L, Cruz R, Rojas Z, et al. Prevalencia y factores de riesgo del consumo de cigarrillo para estudiantes universitarios entre 18 a 25 años, durante el primer semestre académico del año 2013. Medunab. 2013; 16(1):13-18.

16. Sanchez Z, Opaleye E, Martins S, Ahluwalia J, Noto A. Adolescent gender differences in the determinants of tobacco smoking: a cross sectional survey among high school students in São Paulo. BMC Public Health. 2010; 10(12):748-57.

17. Betancour A, Zambrano R. Prevalencia del consumo de tabaco en una muestra de adolescentes escolarizados del municipio de Ebéjico, departamento de Antioquia, Colombia. Liber. 2014;20(1):131-9.

18. Vega A, Aramendi P. La mediación educativa de los programas de cualificación profesional inicial: a propósito de las drogas. Educación XXI. 2011;14(2): 213-36.

19. García O, Suárez R, Santonja F, Secades R, Sánchez E. Psychosocial risk factors for adolescent smoking: A school-based study. International Journal of Clinical and Health Psychology. 2011;11(1):23-33.

20. Iglesias V, Cavada G, Silva C, Cáceres D. Consumo precoz de tabaco y alcohol como factores modifi cadores del riesgo de uso de marihuana. Rev Saúde Pública. 2007; 41(4):517-22. 